\title{
A Study of Lattice and Magnetic Interactions of Conduction Electrons
}

\author{
Hanno Essén \\ Department of Mechanics \\ Royal Institute of Technology \\ S-100 44 Stockholm, Sweden
}

1994, April 27

Telephone: +468 7908759

Fax number: +46 87969850

e-mail: hanno@mech.kth.se

Appeared in: Physica Scripta 52, pp.388-394 (1995) 


\begin{abstract}
Using basic theoretical considerations we show that the elastic continuum and conduction electrons interact via a vector field, to first order. This interaction must therefore be closely related to a magnetic interaction. We then study the magnetic interaction between conduction electrons using the Darwin approach and the free electron gas model. We show that pairing of conduction electrons may result and we calculate the total energy lowering due to the Darwin term in the Hamiltonian. The relevance of the results to superconductivity is considered.
\end{abstract}

\title{
1 Introduction
}

The free (Fermi) electron gas is a very successful model that, suitably perturbed, explains the essential features of ordinary conductivity and resistivity in metals [1]. In the case of resistivity the perturbation is scattering of electrons by thermal fluctuations of the crystal lattice. When the temperature goes down these fluctuations go to zero. Empirically three different things may then happen: Either the free electron gas model remains good and the resistivity goes down all the way to absolute zero, or some other perturbation becomes important and a phase transition occurs. These are of two types magnetic and superconducting.

In the magnetic case these perturbations are well known to be related to electron spin interactions. What other perturbing influences can be contemplated? In many-electron problems the most important perturbation of the independent electron model is, usually, Coulomb correlation. Since the basic idea here is that the electrons are free it does not seem consistent to consider the higher order correlation effects. Kohn and Luttinger [2] have shown that if long range Coulomb correlation is responsible for superconductivity at all, the transition temperature would be very small.

What perturbations remain? Essentially two. The standard candidate for superconductivity is the breakdown of the Born-Oppenheimer separation of electronic and nuclear (lattice) motion. The thermal fluctuations which cause resistivity result in such breakdown, but there is also a non-thermal interaction of the electronic degrees of freedom with the classical elastic displacement (vector) field. In the first part of this article we discuss how this 
interaction must look, to first order, and we find that it is closely analogous to the magnetic interaction. For example, only the transverse part of the elastic displacement field is of importance. The main difference is that it is difficult to determine the coupling strength.

There are also the higher order electromagnetic (or relativistic) interactions between the electrons which can not be expected to be screened in the way the Coulomb repulsion is. These first order correction terms to charged particle interaction were first derived by Darwin [3] and will be discussed in the second part of this article. We first show that this interaction will lead to a bound state for the relative motion of two electrons, if they have (almost) the same unpaired momenta. That is, we get pairing. We then calculate the effect of the Darwin term on the Fermi gas as a whole. Only electrons with unpaired momenta on the Fermi surface will be contribute, but it is shown that this is enough to result in an energy lowering per electron of roughly $10^{-4}$ of the Fermi energy. This is a typical energy gap in ordinary superconductors.

The conclusion is that both perturbations cause an attractive interaction between parallel currents. Because of the Pauli exclusion principle electrons at the Fermi surface have considerable kinetic energy and thus a considerable current will result if the distribution of electrons on the Fermi surface is not isotropic, without cost of kinetic energy. Thus the minimum energy ground state might be one one with flow of current.

\section{The Electron Lattice Interaction}

The main idea here is that the interaction between the conduction electrons and the crystal lattice approximately can be understood in terms of a classical field. The reason for this is that, to a first approximation, the conduction electrons are free delocalized non-interacting particles. They are thus not strongly affected by the detailed atomic structure of the metal but only by long range average properties. In standard solid state jargon the electrons are usually described as being scattered by phonons, the quanta associated with lattice vibrations. Just as most aspects of the interaction of matter with the electromagnetic field do not require the photon concept, but can be understood in terms of a classical field, it should be possible to understand many aspects of conduction electron lattice interaction in terms of the clas- 
sical displacement field. This is a (classical) vector field which describes the deformation of the crystal in the continuum, elastic, limit.

The basis for the separation of the electronic degrees of freedom from the nuclear motion is the so called Born-Oppenheimer approximation. The total Lagrangian for the system is

$$
L=L_{0}+L_{\mathrm{int}}=\left(L_{\mathrm{el}}+L_{\mathrm{nu}}\right)+L_{\mathrm{int}},
$$

and if the interaction $L_{\text {int }}$ is small and can be neglected the Born-Oppenheimer approximation results. Though the separation, in principle, is symmetric [4], one finds that, in molecules, the nuclear part will depend on the electronic state. In metals this is clearly not the case: the vibrational elastic properties do not depend much on the precise state of the conduction electrons, and vice versa. Thus in a metal the elastic and the (conduction) electronic degrees of freedom really separate (symmetrically) in a first approximation.

In the next subsection we discuss the dynamics of the (nuclear) elastic degrees of freedom and the displacement (vector) field. We then proceed to investigate what $L_{\text {int }}$ must look like, in a first approximation.

\subsection{Dynamics of the Displacement Field}

We consider the conduction electrons as moving in a vacuum, albeit a vacuum with properties rather different from the vacuum of empty space. The displacement field is a vector field and we denote it $\boldsymbol{u}$, and its three components by $u_{i}$. As all vector fields it can be decomposed, according to Helmholtz, into a rotation-free, longitudinal part, $\boldsymbol{u}^{\mathrm{L}}$, and a divergence-free, transverse part, $\boldsymbol{u}^{\mathrm{T}}$, (see e.g. Fetter and Walecka [5])

$$
\boldsymbol{u}=\boldsymbol{u}^{\mathrm{L}}+\boldsymbol{u}^{\mathrm{T}},
$$

where

$$
\nabla \times \boldsymbol{u}^{\mathrm{L}}=\mathbf{0} \text { and } \nabla \cdot \boldsymbol{u}^{\mathrm{T}}=0
$$

These can then be written

$$
\begin{aligned}
\boldsymbol{u}^{\mathrm{L}}=\nabla \Theta & \Longleftrightarrow u_{i}^{\mathrm{L}}=\partial_{i} \Theta \\
\boldsymbol{u}^{\mathrm{T}}=\nabla \times \boldsymbol{C} & \Longleftrightarrow u_{i}^{\mathrm{T}}=e_{i j k} \partial_{j} C_{k} .
\end{aligned}
$$

A constant displacement field will only represent a translation of the crystal as a whole. Therefore only derivatives of the displacement field will represent 
elastic deformation. The antisymmetric combination, however, represents rigid rotation, so only the symmetric combination is of interest:

$$
u_{i j}=\frac{1}{2}\left(\partial_{i} u_{j}+\partial_{j} u_{i}\right)
$$

This is the strain tensor in the linear approximation. In the same linear (Hookean) approximation the elastic energy of deformation per unit volume of the crystal is

$$
\mathcal{V}_{\mathrm{nu}}=\frac{1}{2} \lambda_{i j k l} u_{i j} u_{k l}
$$

where $\lambda_{i j k l}$ is the elastic modulus, or stiffness, tensor. This tensor can be taken to have the symmetries

$$
\lambda_{i j k l}=\lambda_{j i k l}=\lambda_{i j l k}=\lambda_{k l i j},
$$

see e.g. Landau and Lifshitz [6].

The kinetic energy per unit volume of the displacement vector field is given by

$$
\mathcal{T}_{\mathrm{nu}}=\frac{1}{2} \varrho_{\mathrm{m}} \dot{u}_{i} \dot{u}_{i}
$$

where $\varrho_{\mathrm{m}}$ is the mass-density, and we thus take the Lagrangian density of the displacement field to be

$$
\mathcal{L}_{\mathrm{nu}}=\frac{1}{2} \varrho_{\mathrm{m}} \dot{u}_{i} \dot{u}_{i}-\frac{1}{2} \lambda_{i j k l} u_{i j} u_{k l} .
$$

This determines the dynamics of the vector field $\boldsymbol{u}$ when conduction electron interaction is neglected.

\subsection{Electron Displacement Field Interaction}

The unperturbed Lagrangian of the free conduction electrons is

$$
L_{\mathrm{el}}=\sum_{i} \frac{1}{2} m \boldsymbol{v}_{i}^{2}
$$

The interaction of these electrons with the vector field $\boldsymbol{u}$ must be a scalar. Only two scalars arise naturally, $\boldsymbol{v}_{i} \cdot \boldsymbol{u}$ and $\Theta$. We thus take the interaction terms to be given by

$$
L_{\mathrm{int}}=\sum_{i}\left[\alpha \boldsymbol{v}_{i} \cdot \boldsymbol{u}\left(\boldsymbol{r}_{i}\right)+\beta \Theta\left(\boldsymbol{r}_{i}\right)\right]
$$


Here $\alpha$ and $\beta$ are some effective coupling constants, presumably proportional to the charge of the electron, since physically the lattice electron interaction is electrostatic in nature. One now notes that only the transverse part of $\boldsymbol{u}$ contributes to the interaction since

$$
\boldsymbol{v} \cdot \boldsymbol{u}^{\mathrm{L}}=\frac{\mathrm{d} \boldsymbol{r}}{\mathrm{d} t} \cdot \nabla \Theta=\frac{\mathrm{d} \Theta}{\mathrm{d} t}
$$

is a total time-derivative and does not affect the dynamics. One also notes that while $L_{\text {nu }}$ contains the first derivatives of $\boldsymbol{u}^{\mathrm{T}}$, it contains $\Theta$ only through second derivatives. The importance of $\Theta$ for the interaction on large length scales must therefore be smaller than that of $\boldsymbol{u}^{T}$. The interaction part of the Lagrangian can thus, to first approximation, be taken as

$$
L_{\text {int }}=\sum_{i} \alpha \boldsymbol{v}_{i} \cdot \boldsymbol{u}^{\mathrm{T}}
$$

The role of the transverse displacement field is thus seen to be closely analogous to the role of the vector potential in the electromagnetic case. In that case the interaction is well known to be $\sim e\left(\boldsymbol{v}_{i} / c\right) \cdot \boldsymbol{A}$.

The Lagrangian for the full system of electrons and elastic continuum is thus seen to be

$$
L=L_{\mathrm{el}}+L_{\mathrm{nu}}+L_{\mathrm{int}}=\sum_{i} \frac{1}{2} m \boldsymbol{v}_{i}^{2}+\int\left(\mathcal{T}_{\mathrm{nu}}-\mathcal{V}_{\mathrm{nu}}\right) \mathrm{d} V+\sum_{i} \alpha \boldsymbol{v}_{i} \cdot \boldsymbol{u}^{\mathrm{T}}
$$

The corresponding Hamiltonian is then

$$
H=\sum_{i} \frac{1}{2 m}\left(\boldsymbol{p}_{i}-\alpha \boldsymbol{u}^{\mathrm{T}}\right) \cdot\left(\boldsymbol{p}_{i}-\alpha \boldsymbol{u}^{\mathrm{T}}\right)+\int\left(\mathcal{T}_{\mathrm{nu}}+\mathcal{V}_{\mathrm{nu}}\right) \mathrm{d} V
$$

and here too one sees that the interaction with the lattice is similar to magnetic interactions. Also the displacement field Lagrangian $L_{\mathrm{nu}}$ is a positive definite quadratic form in the first derivatives of $\boldsymbol{u}^{T}$, though interaction with (first derivatives of) $\boldsymbol{u}^{\mathrm{L}}$ in it makes things a bit complicated. If these can be neglected one can expect the field equation for $\boldsymbol{u}^{\mathrm{T}}$ to be an elliptic partial differential equation with electron current as source.

Just as the Darwin Hamiltonian (18), to be considered below, comes from a full Hamiltonian for electrons plus magnetic field, a similar set of approximations can be applied to the theory described by (16). I. e. we 
might assume that the independent degrees of freedom of the field $\boldsymbol{u}^{\mathrm{T}}$ are not important; only the indirect effect that causes an electron-electron interaction (virtual phonons) need be considered to first order. It then seems clear that the result must be an attraction between parallel currents, just as in the Darwin case.

Onset of superconductivity has been observed to be correlated to changes in the lattice dynamics by Testardi et al. [7], and this may be considered as evidence that the type of coupling of electronic and elastic degrees of freedom, discussed above, can be relevant to superconductivity. Such direct evidence is, however, not common so any change in lattice dynamics must normally be quite small.

Concerning the strength of the coupling one notes that the velocity factor, $\left(v_{\mathrm{F}} / c\right)^{2}$, which limits the strength of magnetic interactions, will not be a problem in the elastic displacement field case. In this case the relevant electron velocity, will still be the Fermi velocity, $v_{\mathrm{F}}$, but $c$ must be replaced by the speed of transverse acoustic waves, $c_{\mathrm{T}}$, and thus make this factor more favorable (by ten orders of magnitude). The actual coupling constant, $\alpha$, on the other hand, is difficult to predict without more detailed considerations, but it is clearly quite small.

Below, equation (40), we estimate the magnetic interaction between electrons to give to an energy lowering per electron which is roughly

$$
\Delta_{\mathrm{D}} \sim R_{0} k_{\mathrm{F}} \mathcal{E}_{\mathrm{F}}=\frac{1}{2} e^{2}\left(\frac{v_{\mathrm{F}}}{c}\right)^{2} k_{\mathrm{F}} .
$$

If the present mechanism is to have some chance of explaining high-temperature superconductivity, one must make this Darwin energy lowering per electron larger by two orders of magnitude. In view of the improved velocity factor, $\left(v_{\mathrm{F}} / c_{\mathrm{T}}\right)^{2} \sim 10^{10}\left(v_{\mathrm{F}} / c\right)^{2}$, one sees that it is enough if the 'effective charge' in the elastic coupling is roughly $10^{-4}$ of the electron charge. One should also keep in mind that in the complicated crystals that exhibit hightemperature superconductivity there may be other (structural) vector-fields, apart from the overall elastic displacement field, that couple to the electron motion in the manner outlined here. 


\section{Effect of Darwin Attraction between Con- duction Electrons}

Relativistic corrections to the dynamics of the conduction electrons are normally not considered in superconductivity theory, it being taken for granted that these are too small to be of relevance in comparison with perturbations from phonons and other effects. The relevant parameter determining their order of magnitude is $\left(v_{\mathrm{F}} / c\right)^{2}$, where $v_{\mathrm{F}}$ is the Fermi velocity and $c$ the speed of light. This number is, however, of order of magnitude $10^{-4}$ and it is thus not obvious that they are totally negligible. For example the energy gap in superconductors is typically $10^{-4} \mathcal{E}_{\mathrm{F}}$ so for this purpose this interaction has just the right strength.

\subsection{The Darwin Hamiltonian}

Darwin [3] derived the first order relativistic corrections to the Lagrangian for charged particles in 1920. That these relativistic terms can be of importance even in ordinary macroscopic physics when magnetic phenomena are considered has been shown by Coleman and Van Vleck [8]. Physically the approximation arises from the full Lagrangian of particles plus electromagnetic fields when the independent degrees of freedom of the fields are neglected. This corresponds to radiation being negligible so that there are no (non-virtual) photons present.

The Hamiltonian for a system of electrons, including Darwin's first order relativistic corrections, is according to Landau and Lifshitz [9] (we ignore spin)

$$
H=\sum_{i} \frac{\boldsymbol{p}_{i}^{2}}{2 m}-\sum_{i} \frac{\boldsymbol{p}_{i}^{4}}{8 c^{2} m^{3}}+\sum_{i<j} \frac{e^{2}}{r_{i j}}-\sum_{i<j} \frac{e^{2}\left[\boldsymbol{p}_{i} \cdot \boldsymbol{p}_{j}+\left(\boldsymbol{p}_{i} \cdot \boldsymbol{e}_{i j}\right)\left(\boldsymbol{p}_{j} \cdot \boldsymbol{e}_{i j}\right)\right]}{2 c^{2} m^{2} r_{i j}} .
$$

Here the second term reduces the kinetic energy compared to the classical value, but does not lead to qualitatively interesting changes of the dynamics, so we ignore it. The Coulomb repulsion is strongly screened in metals; from the point of view of a conduction electron the rest of the metal has charge $+e$ and this positive charge must be localized near the electron, since any other distribution would be energetically very unfavorable. This is the main reason that the free electron approximation is so successful as a model for the 
conduction electrons [1]. Our main interest will be in conduction electrons near the Fermi energy, and we assume these are so far apart that the screened Coulomb interaction can be neglected. We are thus left with the first and the last terms in our Hamiltonian (18).

The last term corresponds to the interaction of currents and shows that parallel currents attract each other. In classical electrodynamics this is a well known effect and is sometimes referred to as the 'pinch' effect. The corresponding contribution to the energy for a current distribution $\boldsymbol{j}(\boldsymbol{r})$ is

$$
-\frac{1}{4 c^{2}} \iint\left(\frac{\boldsymbol{j}(\boldsymbol{r}) \cdot \boldsymbol{j}\left(\boldsymbol{r}^{\prime}\right)}{\left|\boldsymbol{r}-\boldsymbol{r}^{\prime}\right|}+\frac{\boldsymbol{j}(\boldsymbol{r}) \cdot\left(\boldsymbol{r}-\boldsymbol{r}^{\prime}\right) \boldsymbol{j}\left(\boldsymbol{r}^{\prime}\right) \cdot\left(\boldsymbol{r}-\boldsymbol{r}^{\prime}\right)}{\left|\boldsymbol{r}-\boldsymbol{r}^{\prime}\right|^{3}}\right) \mathrm{d} V \mathrm{~d} V^{\prime} .
$$

The second term has the effect that electrons moving one after the other attract twice as much as electrons moving side by side. The (spherical) average effect of the two terms is given by

$$
-\frac{1}{3 c^{2}} \iint \frac{\boldsymbol{j}(\boldsymbol{r}) \cdot \boldsymbol{j}\left(\boldsymbol{r}^{\prime}\right)}{\left|\boldsymbol{r}-\boldsymbol{r}^{\prime}\right|} \mathrm{d} V \mathrm{~d} V^{\prime}
$$

It is thus not obvious that the ground state of a system must correspond to $\boldsymbol{j}=\mathbf{0}$. Classically the kinetic energy associated with motion will give a positive contribution that outweighs this term, but in quantum mechanics the Pauli principle forces electrons to have large velocities even in the ground state, so the possibility of a macroscopic (or mesoscopic) current distribution in the ground state of a metal cannot be ruled out on theoretical grounds.

Heisenberg, long ago, suggested that current flows in the superconducting ground state [10]. The mechanism suggested by Heisenberg was, however, not convincing. A ground state current makes the zero resistance property of superconductors easier to understand. Their other main property, the Meissner effect, or complete diamagnetism, can be understood as due to an energy gap above the Fermi level as originally outlined by Welker [11], who, however did not explain the origin of the gap. Welker [12] also originally suggested that the magnetic attraction of currents might be responsible for superconductivity. Unfortunately he did not calculate the ground state energy properly and thus early attempts in this direction ended in confusion. Part of the motivation for the present work is to once again consider these possibilities. 


\subsection{Electron Pairing}

Consider two electrons, in a metal, interacting via the Darwin term. This, of course, means that we assume that all other electrons have paired momenta and do not contribute to the current density. We neglect the uninteresting correction to the kinetic energy and the screened Coulomb repulsion and take the Hamiltonian for these to be (see equation (18))

$$
H=\frac{\boldsymbol{p}_{1}^{2}}{2 m}+\frac{\boldsymbol{p}_{2}^{2}}{2 m}-\frac{e^{2}}{2 m^{2} c^{2}} \frac{\left[\boldsymbol{p}_{1} \cdot \boldsymbol{p}_{2}+\left(\boldsymbol{p}_{1} \cdot \boldsymbol{e}_{12}\right)\left(\boldsymbol{p}_{2} \cdot \boldsymbol{e}_{12}\right)\right]}{r_{12}} .
$$

We now make the (canonical) transformation

$$
\begin{array}{ll}
\boldsymbol{P}=\frac{1}{\sqrt{2}}\left(\boldsymbol{p}_{1}+\boldsymbol{p}_{2}\right), & \boldsymbol{p}=\frac{1}{\sqrt{2}}\left(\boldsymbol{p}_{1}-\boldsymbol{p}_{2}\right), \\
\boldsymbol{R}=\frac{1}{\sqrt{2}}\left(\boldsymbol{r}_{1}+\boldsymbol{r}_{2}\right), & \boldsymbol{r}=\frac{1}{\sqrt{2}}\left(\boldsymbol{r}_{1}-\boldsymbol{r}_{2}\right), \\
\boldsymbol{p}_{1}=\frac{1}{\sqrt{2}}(\boldsymbol{P}+\boldsymbol{p}), & \boldsymbol{p}_{2}=\frac{1}{\sqrt{2}}(\boldsymbol{P}-\boldsymbol{p}) .
\end{array}
$$

The Hamiltonian then becomes

$$
H=\frac{\boldsymbol{P}^{2}}{2 m}+\frac{\boldsymbol{p}^{2}}{2 m}-\frac{e^{2}}{4 \sqrt{2} m^{2} c^{2}} \frac{\boldsymbol{P}^{2}+(\boldsymbol{P} \cdot \boldsymbol{e})^{2}}{r}+\frac{e^{2}}{4 \sqrt{2} m^{2} c^{2}} \frac{\boldsymbol{p}^{2}+(\boldsymbol{p} \cdot \boldsymbol{e})^{2}}{r} .
$$

Here $\boldsymbol{e}=\boldsymbol{e}_{12}=\left(\boldsymbol{r}_{1}-\boldsymbol{r}_{2}\right) /\left|\boldsymbol{r}_{1}-\boldsymbol{r}_{2}\right|$ is the unit vector from electron 2 to electron 1 .

For two electrons, at the Fermi surface, with (nearly) the same momentum and opposite spin we can make the ansatz $\left(|\boldsymbol{k}|=k_{\mathrm{F}}\right.$, the Fermi wave number, see Appendix)

$$
\Psi(\boldsymbol{R}, \boldsymbol{r})=\frac{1}{L^{3}} \exp \left(\mathrm{i} \boldsymbol{k} \cdot \boldsymbol{r}_{1}\right) \exp \left(\mathrm{i} \boldsymbol{k} \cdot \boldsymbol{r}_{2}\right) \Phi(\boldsymbol{r})=\frac{1}{L^{3}} \exp \left(\frac{\mathrm{i}}{\hbar} \boldsymbol{P} \cdot \boldsymbol{R}\right) \Phi(\boldsymbol{r})
$$

where the function $\Phi$ is symmetric and describes the relative motion of the two electrons. If we assume that the relative momentum, $\boldsymbol{p}$, is small compared to the common momentum, $\boldsymbol{P} / \sqrt{2}=\hbar \boldsymbol{k}$, we can neglect the last term in the Hamiltonian (23), and find that the relative wave function, $\Phi$, obeys the Schrödinger equation

$$
\left(-\frac{\hbar^{2}}{2 m} \nabla^{2}-\frac{\hbar^{2} e^{2} k_{\mathrm{F}}^{2}}{2 \sqrt{2} m^{2} c^{2}} \frac{1+\cos ^{2} \vartheta}{r}\right) \Phi=E \Phi .
$$


Here $\vartheta$ is the angle between the vector $\boldsymbol{r}$ and $\boldsymbol{k}$. The long range (Coulomb) nature of the interaction shows that the ground state for the relative motion of the two electrons will be bound. Thus a single pair of electrons at the Fermi surface will form a bound state when they move in the same direction. The fact that the interaction is weak does not matter since it is long range. Classically the interaction corresponds to a magnetic interaction and magnetic fields are known to be badly screened by metals, except in the superconducting state.

To estimate the properties of this bound state quantitatively we first replace $1+\cos ^{2} \vartheta$ by its spherical average: $1+\overline{\cos ^{2} \vartheta}=4 / 3$. If we now replace $4 /(3 \sqrt{2})=0.943$ by one, we find that the interaction potential in the Schrödinger equation (25) is roughly

$$
V(r) \approx-\frac{\mathcal{E}_{\mathrm{F}} R_{0}}{r}
$$

where $R_{0} \equiv e^{2} /\left(m c^{2}\right)$ is the classical electron radius and $\mathcal{E}_{\mathrm{F}} \equiv \hbar^{2} k_{\mathrm{F}}^{2} /(2 m)$ is the Fermi energy. The corresponding Bohr-radius is then (the 'DarwinFermi'-radius)

$$
R_{\mathrm{DF}}=\frac{\hbar^{2}}{m \mathcal{E}_{\mathrm{F}} R_{0}}=\frac{m c^{2}}{\mathcal{E}_{\mathrm{F}}} \frac{\hbar^{2}}{m e^{2}}=\frac{m c^{2}}{\mathcal{E}_{\mathrm{F}}} r_{\mathrm{B}}
$$

where $r_{\mathrm{B}}$ is the ordinary Bohr-radius. Since $m c^{2}=5.11 \cdot 10^{5} \mathrm{eV}$ while $\mathcal{E}_{\mathrm{F}}$ typically is $10 \mathrm{eV}$ we find that

$$
R_{\mathrm{DF}} \sim 5 \cdot 10^{4} r_{\mathrm{B}}
$$

The ground state energy can be estimated to be

$$
E_{\mathrm{DF}}=-\frac{1}{2} \frac{m\left[e^{2}\left(\frac{\mathcal{E}_{\mathrm{F}}}{m c^{2}}\right)\right]^{2}}{\hbar^{2}} \sim 4 \cdot 10^{-10} E_{\mathrm{H}},
$$

where $E_{\mathrm{H}}$ is the ground state energy of the Hydrogen atom. This is an extremely small energy. In the next section we will see how conduction electrons can collaborate to get much lower energy.

\subsection{Ground State Darwin Energy}

We now consider the effect of the last term in the Hamiltonian (18) considered as a perturbation on free (conduction) electrons. Assuming a Hartree wave 
function, only the electrons at the Fermi surface will contribute since those inside are paired and have no net current density. The electrons are thus considered to be in states

$$
\psi_{i}(\boldsymbol{r})=\frac{1}{\sqrt{L^{3}}} \exp \left(\mathrm{i} \boldsymbol{k}_{i} \cdot \boldsymbol{r}\right)
$$

with $\left|\boldsymbol{k}_{i}\right|=k_{\mathrm{F}}$. To find the expectation value of the Darwin term we need the electrostatic interaction energy of two electrons with constant charge density within a cubic box of volume $L^{3}$. This quantity can be written $\kappa e^{2} / L$ where $\kappa$ is the dimensionless constant given by

$$
\kappa \equiv \int_{\Omega} \int_{\Omega} \frac{\mathrm{d} V_{1} \mathrm{~d} V_{2}}{\left|\boldsymbol{r}_{1}-\boldsymbol{r}_{2}\right|} \approx 1.882312645,
$$

where the integrations are over the unit cube [13].

The Darwin energy of the $N_{\mathrm{c}}$ electrons on the Fermi surface is then found to be

$$
E_{\mathrm{D}}=-\frac{\hbar^{2}}{2 c^{2} m^{2}} \frac{\kappa e^{2}}{L} \sum_{i<j}^{N_{\mathrm{c}}} \boldsymbol{k}_{i} \cdot \boldsymbol{k}_{j} .
$$

We have ignored exchange.

The perturbation energy can be written

$$
E_{\mathrm{D}}=-\mathcal{E}_{\mathrm{F}} R_{0}\left(\frac{\kappa}{L}\right) \sum_{i<j}^{N_{\mathrm{c}}} \cos \theta_{i j} .
$$

Here $\theta_{i j}$ is the angle between the directions of the two wave vectors $\boldsymbol{k}_{i}$ and $\boldsymbol{k}_{j}$. Clearly the sum of the scalar products is zero if the distribution of the wave vectors is isotropic on the Fermi surface (sphere), since then $\cos \theta_{i j}$ must be positive as often as negative. The value of this perturbation thus depends essentially on how anisotropic this distribution can become. This, in turn, depends on the ratio, $\gamma$, of the number of electrons, $N_{\mathrm{c}}$, and number of states, $N_{\mathrm{s}}$, on the Fermi sphere. This number is (see Appendix)

$$
\gamma \equiv \frac{N_{\mathrm{c}}}{N_{\mathrm{s}}} \text { where, } N_{\mathrm{s}}=\frac{2 L^{2} k_{\mathrm{F}}^{2}}{\pi},
$$

and it must obey $0<\gamma<1$. The most anisotropic distribution of $\boldsymbol{k}$-vectors on the Fermi sphere corresponds to these all falling within a solid angle 
$\Omega=\gamma 4 \pi$ around some arbitrary direction. Using this we can approximate the sum of the cosines as follows

$$
\sum_{i<j}^{N_{\mathrm{c}}} \cos \theta_{i j} \approx \frac{1}{2} N_{\mathrm{s}}^{2} \gamma^{2} \cos (\sqrt{\gamma} \pi / 2) .
$$

Here we have used that the number of terms in the sum is roughly $N_{\mathrm{c}}^{2} / 2=$ $N_{\mathrm{s}}^{2} \gamma^{2} / 2$ and that the average value of $\theta_{i j}$ must be proportional to the square root of the solid angle, i.e. to $\sqrt{\gamma}$. The proportionality factor has been chosen so that the average angle is $\pi / 2$ for $\gamma=1$, as it should. If we now put

$$
\chi(\gamma) \equiv \gamma^{2} \cos (\sqrt{\gamma} \pi / 2)
$$

we can write the perturbation energy, equation (33), as follows

$$
E_{\mathrm{D}} \approx-\mathcal{E}_{\mathrm{F}} R_{0}\left(\frac{\kappa}{L}\right) \frac{1}{2} N_{\mathrm{s}}^{2} \chi(\gamma)
$$

In order to estimate the maximum size of this energy we note that the function $\chi(\gamma)$ has its maximum at $\gamma_{\max } \approx 0.648$ and that the value of this maximum is $\chi_{\max } \equiv \chi\left(\gamma_{\max }\right) \approx 0.127$ (see figure 1 ). If we also insert the value for the number of states at the Fermi surface, from equation (34), we get

$$
E_{\mathrm{D}} \approx-\mathcal{E}_{\mathrm{F}} R_{0}\left(\frac{2 \kappa \chi_{\max }}{\pi^{2}}\right) k_{\mathrm{F}}^{4} L^{3}
$$

for the total 'Darwin' energy of the conduction electrons. One should note that it is only the last term in the Hamiltonian (18) that is sensitive to the distribution of $\boldsymbol{k}$-vectors on the Fermi-sphere. The other terms have thus not been neglected; they are constant under variation of this distribution.

One notes that this energy is proportional to the volume as it should be ('size consistency'). Normally one would expect the effect of the long range Coulomb-like interaction to diverge for some constant bulk current density, see equation (20). The fact that this does not happen is due to the two-dimensionality of the Fermi surface. Quantum mechanics thus plays two roles here. The Pauli principle forces electrons to have large speeds, even in the ground state so, in principle, the ground state might be one with macroscopic currents. If a three dimensional bulk current density was possible its (negative) energy contribution would diverge as the size of the 
sample increased. The Pauli principle, however, also restricts the mobile electrons to a two-dimensional surface (in momentum space) and thus this divergence is prevented. This fact was not discovered by Welker [12] and thus early attempts in this direction stranded on what was called the "magnetic catastrophe".

\subsection{Darwin Energy per Conduction Electron}

The total Darwin energy, found above using first order perturbation theory, does not say anything about the spectrum of the conduction electrons. It is wrong, however, to assume that only the electrons at the Fermi surface (which are responsible for the energy lowering) will be affected. The energy gain due to the electrons at the Fermi surface distributes itself among all conduction electrons since these are identical particles and their orbitals must remain orthogonal. Exactly how it distributes itself among the conduction electrons we don't know. To get a rough estimate it seems reasonable to assume that it is distributed evenly among them. This is assumed below.

We now calculate the Darwin energy per conduction electron. We get this energy lowering per electron, $\Delta_{\mathrm{D}}$, by multiplying $E_{\mathrm{D}} / L^{3}$ by $L^{3} / N=1 / n$ where $n$, given by (see Appendix)

$$
n \equiv \frac{N}{L^{3}}=\frac{1}{3 \pi^{2}} k_{\mathrm{F}}^{3}
$$

is the number density of conduction electrons. The result is

$$
\Delta_{\mathrm{D}} \equiv-\frac{E_{\mathrm{D}}}{N} \approx\left(6 \kappa \chi_{\max }\right) R_{0} k_{\mathrm{F}} \mathcal{E}_{\mathrm{F}} \approx 1.43 \cdot R_{0} k_{\mathrm{F}} \mathcal{E}_{\mathrm{F}}
$$

In atomic units the classical electron radius is $R_{0}=5.3 \cdot 10^{-5}$ so, collecting numbers, we get that

$$
\Delta_{\mathrm{D}} \approx\left(7.6 \cdot 10^{-5}\right) k_{\mathrm{F}} \mathcal{E}_{\mathrm{F}} .
$$

Since $k_{\mathrm{F}}$ is roughly one ( $k_{\mathrm{F}} \sim 1$ in atomic units) for many metals we see that this predicts the gap energy to be roughly $\Delta_{\mathrm{D}} \sim 10^{-4} \mathcal{E}_{\mathrm{F}}$, assuming, of course, that we can identify the energy lowering per electron with the energy gap (at zero temperature).

There is in fact good agreement between $\Delta_{\mathrm{D}}$ and the smaller experimental energy gaps. For example, for Zn formula (41) gives $\Delta_{\mathrm{D}}=2.1 \cdot 10^{-5}$ which 
may be compared to the experimental value $1.7 \cdot 10^{-5}$. The corresponding values for $\mathrm{Al}$ are $\Delta_{\mathrm{D}}=3.1 \cdot 10^{-5}$ and $2.5 \cdot 10^{-5}$ which thus indicates both correct order of magnitude and a correct trend. For $\mathrm{Pb}$, however, (41) gives $\Delta_{\mathrm{D}}=2.1 \cdot 10^{-5}$ while the experimental result is $20 \cdot 10^{-5}$ (experimental data are taken from Kittel [14]). One reason for this might be effects that have been neglected in the simple free electron model; e.g. the fact that the Fermi surface is not spherical in real metals should increase the relative importance of the Fermi surface. The largest error is, no doubt, due to the even distribution of the energy lowering among all electrons. It is remarkable that one obtains nearly correct values for the low $T_{\mathrm{c}}$ energy gaps without a single free parameter, even if this clearly is is due to several canceling errors (assuming that the theory is at all relevant).

High temperature superconductivity, on the other hand, can hardly be explained using this theory. One notes that two-dimensionality does not help; it only makes things worse. To get considerably better values than the ones obtained here it is necessary to consider interaction with the lattice as we did above.

\section{Discussion and Conclusions}

The most valuable results of the present investigation are, firstly, the formula for the energy lowering due to the magnetic attraction of parallel currents, $\Delta_{\mathrm{D}} \approx 1.43 \cdot R_{0} k_{\mathrm{F}} \mathcal{E}_{\mathrm{F}}$, and, secondly, the proof that the coupling to the elastic displacement field is very similar to a magnetic interaction. If these findings are of any relevance to the theory of superconductivity, as the author firmly believes, it is clear that these two effects will be difficult to distinguish.

The first part about the electron lattice interaction might partly be regarded as, merely, a different way of deriving the traditional BCS theory $[15,16,17]$, which it, of course, will resemble if put in second quantized form. Note, however that there are differences. In BCS-theory the attraction and the pairing of electrons is between electrons with opposite momenta on the Fermi sphere. Such a phenomenon has no classical analog. Here this attraction and pairing is between electrons with similar momenta and consequently small relative motion. Classically it is not at all difficult to understand that two such electrons may form a bound pair as they move together through the crystal. Another difference is that BCS-theory does not predict a current 
in the ground state and thus has some difficulty in explaining the observed fantastic persistency of superconducting currents. In the present theory this is no problem; the state with a current is simply the ground state. One of the problems with the above approach is the fact that no macroscopic current can flow in a simply connected isolated superconducting specimen. If there is a current distribution in such a specimen it must have the same nature as the velocity distribution in homogeneous turbulence, i.e. have no net momentum or angular momentum.

Since the present view, considered as an explanation of superconductivity, thus is at odds with traditional theory on some points, it is fair to ask, how relevant the present theory is. The point of view of the author is that it is possible that the present theory is irrelevant, but, since it is based on quite conservative and standard theoretical physical methods and arguments, it would at least be interesting to know exactly why it is irrelevant.

\section{A Appendix; the Free Electron Gas, the Fermi Surface and the Maximum Current Density}

To make this paper selfcontained some relationships for the free electron gas are reviewed here (see Madelung [18]). Consider noninteracting, spin 1/2 Fermions, constrained to move in a cubic box of side length $L$. Assuming periodic (Born-von Karman) boundary conditions, the allowed states are

$$
\psi_{i}(\boldsymbol{r})=\frac{1}{\sqrt{L^{3}}} \exp \left(\mathrm{i} \boldsymbol{k}_{i} \cdot \boldsymbol{r}\right)
$$

where the wavenumber vectors $\boldsymbol{k}_{i}$ must obey

$$
\boldsymbol{k}_{i}=\frac{2 \pi}{L}\left(n_{i x}, n_{i y}, n_{i z}\right) \text { with } n_{i x}, n_{i y}, n_{i z}=0, \pm 1, \pm 2 \ldots
$$

These states have energy $\mathcal{E}_{i}=\frac{\hbar^{2} \boldsymbol{k}_{i}^{2}}{2 m}=\frac{h^{2}}{2 m L^{2}}\left(n_{i x}^{2}+n_{i y}^{2}+n_{i z}^{2}\right)$ and the total number of such states with energy less than $\mathcal{E}_{\mathrm{F}}$ (corresponding to the Fermi wavenumber $\left.k_{\mathrm{F}}\right)$ is the volume of the sphere with radius $k_{\mathrm{F}} L /(2 \pi)$. This number is $\frac{4 \pi}{3}\left[k_{\mathrm{F}} L /(2 \pi)\right]^{3}$. Since there can be two electrons in each such 
spatial state the total number of electrons accommodated by states with energy less than the Fermi energy is

$$
N=2 \frac{4 \pi}{3} k_{\mathrm{F}}^{3}\left(\frac{L}{2 \pi}\right)^{3}=\frac{1}{3 \pi^{2}} k_{\mathrm{F}}^{3} L^{3}
$$

The conduction electron density is thus $n=N / L^{3}=k_{\mathrm{F}}^{3} /\left(3 \pi^{2}\right)$.

Consider the surface of the Fermi-sphere in k-space. The area of this surface is $4 \pi k_{\mathrm{F}}^{2}$. The $\mathrm{k}$-space area per state is $(2 \pi / L)^{2}$, so, again with two electrons possible per spatial state, this gives that the maximum possible number of electrons on the Fermi surface is

$$
N_{\mathrm{s}}=2 \frac{4 \pi k_{\mathrm{F}}^{2}}{(2 \pi / L)^{2}}=\frac{2}{\pi} k_{\mathrm{F}}^{2} L^{2}
$$

This means that the number of such states per surface area $\left(8 L^{2}\right)$ of quantization volume is $n_{\mathrm{s}}=k_{\mathrm{F}}^{2} /(4 \pi)$. One might note that the ratio of number of surface states to total number of states is

$$
\frac{N_{\mathrm{s}}}{N}=\frac{6 \pi}{k_{\mathrm{F}} L}
$$

and thus goes to zero in the thermodynamic limit $L \rightarrow \infty$. This is, of course, the reason that these rarely are considered, but as we have seen in section 3.3), the Darwin energy per conduction electron remains finite in this limit, because of its long range nature.

It is of interest to calculate the maximum current density in the free electron gas when the the Fermi surface is half filled $(\gamma=1 / 2)$ with electrons. This occurs when all surface states lie on one hemisphere of the Fermi surface. Each electron contributes a current density

$$
j_{\mathrm{F}}=\frac{1}{L^{3}} e v_{\mathrm{F}}
$$

On the surface element $\mathrm{d} A_{\mathrm{F}}=k_{\mathrm{F}}^{2} 2 \pi \sin \theta \mathrm{d} \theta$ of the Fermi sphere there are

$$
\mathrm{d} N_{\mathrm{s}}=\frac{2 L^{2}}{4 \pi^{2}} \mathrm{~d} A_{\mathrm{F}}
$$

one electron states. The contribution of these to the total current density is

$$
\mathrm{d} J_{\mathrm{F}}(\theta)=j_{\mathrm{F}} \cos \theta \mathrm{d} N_{\mathrm{s}}(\theta) .
$$


Integration of this from zero to pi now gives

$$
J_{\mathrm{F} \max }=\frac{\hbar e k_{\mathrm{F}}^{3}}{2 \pi m L}
$$

for the current density (dimension: current per unit area). This quantity is also seen to go to zero in the thermodynamic limit $L \rightarrow \infty$. It is, however, quite large for normal values of $L$,

$$
L J_{\mathrm{F} \max }=\frac{\hbar e}{2 \pi m r_{\mathrm{B}}^{3}}\left(k_{\mathrm{F}} r_{\mathrm{B}}\right)^{3} \approx 2 \cdot 10^{7}\left(k_{\mathrm{F}} r_{\mathrm{B}}\right)^{3} \mathrm{~A} / \mathrm{m},
$$

and must not be understood as a macroscopic current density. Here $r_{\mathrm{B}}$ is the Bohr-radius. 


\section{References}

[1] Ashcroft, N. W. and Mermin, N. D., Solid State Physics, Saunders College, Philadelphia (1976).

[2] Kohn, W. and Luttinger, J. M., Phys. Rev. Letters 15, 524 (1965).

[3] Darwin, C. G., Phil. Mag. 39, 537 (1920).

[4] Essén, H., International Journal of Quantum Chemistry, XII, 721 (1977).

[5] Fetter, A. L. and Walecka, J. D., Theoretical Mechanics of Particles and Continua, McGraw-Hill Publishing Company, New York (1980).

[6] Landau, L. D. and Lifshitz, E. M., Theory of Elasticity, 2nd edn., Pergamon, Oxford (1970).

[7] Testardi, L. R., Bateman, T. B., Reed W. A. and Chirba, V. G., Phys. Rev. Letters, 15, 250 (1965).

[8] Coleman, S. and Van Vleck, J. H., Phys. Rev. 171, 1370 (1968).

[9] Landau, L. D. and Lifshitz, E. M., The Classical Theory of Fields, 4th edn., Pergamon, Oxford (1975).

[10] Heisenberg, W., Two Lectures, Cambridge at the university press, Cambridge (1949).

[11] Welker, H., Physikalische Zeitschrift, 39, 920 (1938).

[12] Welker, H., Zeitschrift für Physik, 114, 525 (1939).

[13] Nordmark, A., private communication, helped calculate this constant. In earlier versions it was estimated using a spherical box. This gave the value 0.967 and thus underestimated the effect by almost a factor 2 .

[14] Kittel, C., Introduction to Solid State Physics, 5th edn., John Wiley, New York (1976).

[15] Cooper, L. N., Phys. Rev. 104, 1189 (1956). 
[16] Bardeen, J., Cooper, L. N. and Schrieffer, J. R., Phys. Rev. 108, 1175 (1957).

[17] Cooper, L. N., Am. J. Phys. 28, 91 (1960).

[18] Madelung, O., Introduction to Solid State Theory (2nd printing 1981), Springer Verlag, Berlin (1978). 
Figure 1: Plot of the function $\chi(\gamma)=\gamma^{2} \cos (\pi \sqrt{\gamma} / 2)$. 